\title{
Lower limb nodules
}

A 45-year-old Chinese woman with no medical history or regular medications presented with painful nodules on her left shin that progressed to involve her right shin, thighs and lower back over a period of 3 months. There was associated lower limb joints stiffness. Systemic review revealed constitutional symptoms of loss of appetite, night sweats and amenorrhea of 3 months' duration. She also had unintentional weight loss of $7 \mathrm{~kg}$ over the preceding 2 months. Examination demonstrated multiple indurated erythematous to violaceous plaques and nodules over bilateral thighs, calves and shins (Fig.1), associated with bilateral lower limb pitting oedema up to mid-thigh.

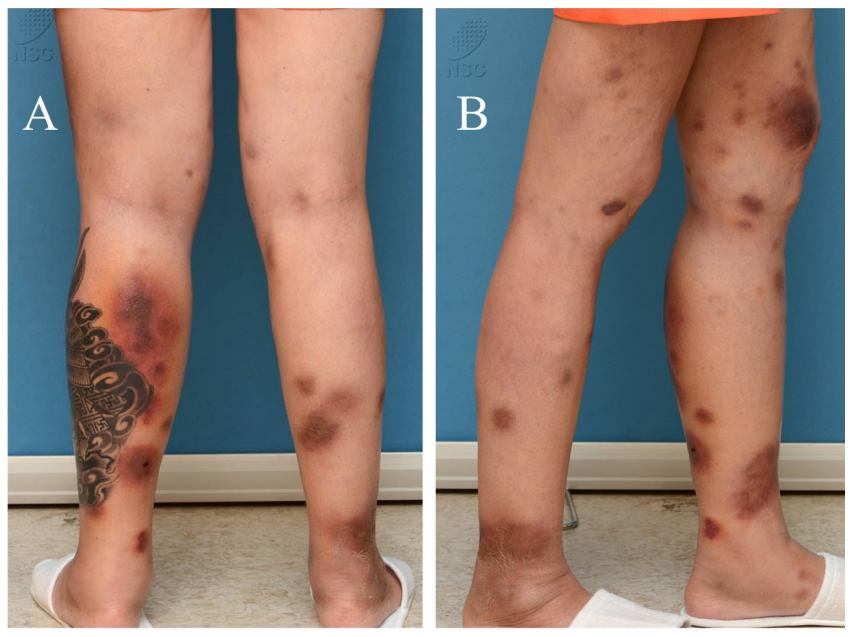

Fig. 1. Indurated erythematous to violaceous plaques and nodules on bilateral lower limbs associated with lower limb oedema from (A) posterior and (B) right lateral views.

Skin biopsy from a right thigh nodule demonstrated a lobular panniculitis. There was extensive necrosis of the subcutis, with a dense neutrophilic infiltrate. Characteristic ghost cells were seen and basophilic calcifications were present (Fig. 2). There was no vasculitis. Ziehl-Neelsen stain was negative for acid-fast bacilli.

Subsequent blood investigations revealed white cell count of $13.21 \times 10^{9} / \mathrm{L}$, eosinophilia at $0.61 \times 10^{9} / \mathrm{L}$, serum lipase of $>400 \mathrm{U} / \mathrm{L}$, normal serum amylase of $47 \mathrm{U} / \mathrm{L}$, raised erythrocyte sedimentation rate of $126 \mathrm{~mm} /$ hour, normal $\mathrm{C} 3$ of $1.56 \mathrm{~g} / \mathrm{L}$, normal $\mathrm{C} 4$ of $0.360 \mathrm{~g} / \mathrm{L}$, negative anti-dsDNA, normal antinuclear antibody of $<80$ titre and normal rheumatoid factor level of $<3.5$. Tumour markers were elevated with CA-125 at $1093 \mathrm{U} / \mathrm{mL}, \mathrm{CA} 19-9$ at $371 \mathrm{U} / \mathrm{mL}$ and alphafetoprotein at $16 \mu \mathrm{g} / \mathrm{L}$. Pan-computed tomography imaging revealed extensive mass lesions along the peritoneum, lesser sac, porta hepatis, omentum and mesentery that were inseparable from the pancreas. There was also a moderate left pleural effusion and indeterminate rounded lymph nodes in the anterior mediastinum and left axilla. Biopsies of the peritoneal and pelvic masses both reported adenocarcinoma on histology.

She was empirically treated with oral prednisolone initially, with only partial improvement of the skin nodules.

What is the most likely diagnosis?
A. Cutaneous sarcoidosis
B. Erythema induratum
C. Erythema nodosum
D. Polyarteritis nodosa
E. Pancreatic panniculitis

Discussion. Various conditions may present clinically with subcutaneous skin nodules. Polyarteritis nodosa is a multisystem vasculitis that often presents with cutaneous involvement, such as palpable nodules and ulcers, with constitutional symptoms and arthritis. Histology characteristically demonstrates segmental necrotising vasculitis of the medium-sized arteries within the deeper dermis and subcutaneous fat. ${ }^{1}$

Sarcoidosis is another multisystem disorder that can present with cutaneous papules and plaques, commonly red-brown in colour, involving the lower limbs and sites of previous injury. Histologically, non-caseating epitheloid cell granulomas are usually observed. ${ }^{2}$

Panniculitides, or disorders of the subcutaneous fat layer, represent another category of conditions that may present as subcutaneous nodules. They may be differentiated by clinical features and the 2 major histologic patterns of panniculitis - septal and lobular.

Erythema nodosum, the most common panniculitis, typically presents with subcutaneous nodules on the anterior aspects of the lower limbs. Histologically, erythema nodosum is the prototypic septal panniculitis, where an inflammatory infiltrate is found primarily within the interlobular fat septae of the subcutis and characteristic Miescher's radial granulomas may be found. ${ }^{3}$ 

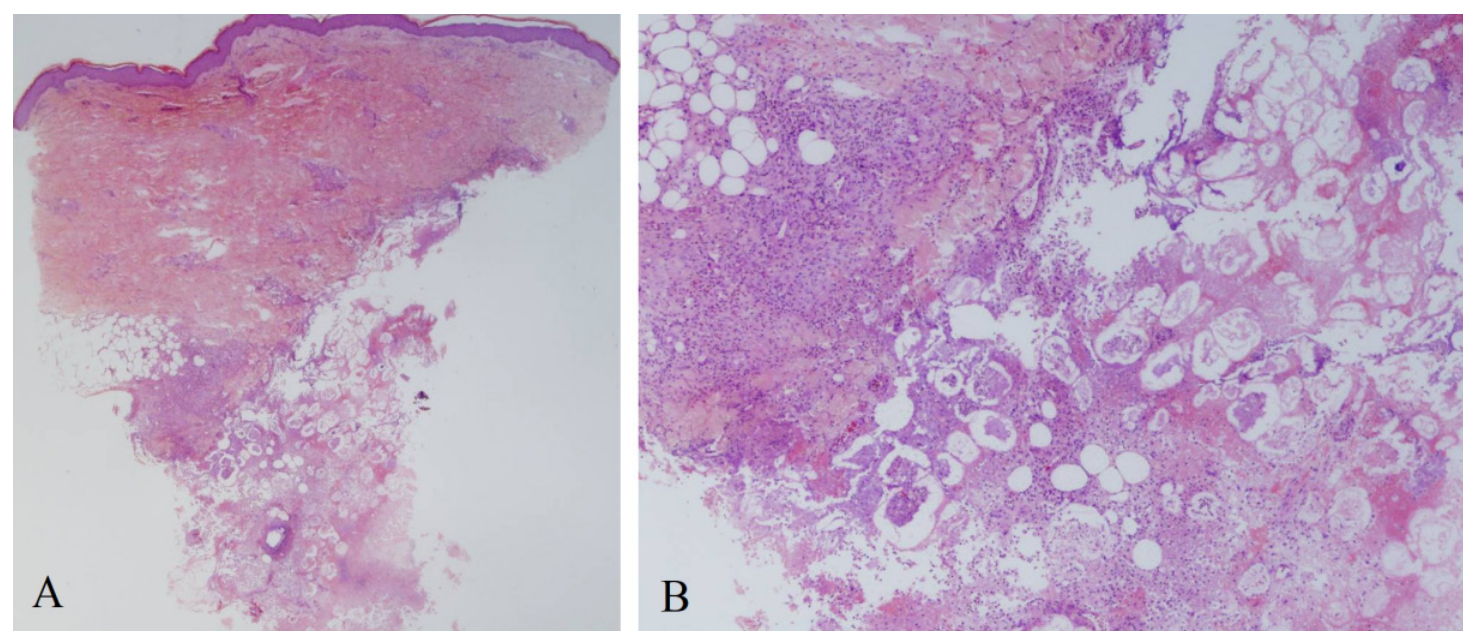

Fig. 2. (A) There is a lobular panniculitis (40x, haematoxylin and eosin) with (B) characteristic ghost cells and basophilic calcification, in association with areas of necrosis and a neutrophilic infiltrate (400x, haematoxylin and eosin).

Erythema induratum, on the other hand, is an uncommon panniculitis that usually presents with subcutaneous nodules on the posterior lower limbs, and is associated with mycobacteria tuberculosis infections. ${ }^{3}$ Histologically, it is a septo-lobular panniculitis with granulomatous inflammation, fat necrosis and associated acute vasculitis. ${ }^{3}$

Other causes of predominantly lobular panniculitis are lupus panniculitis and subcutaneous panniculitislike T-cell lymphoma (SPTCL). Lupus panniculitis usually presents with nodules and plaques on the face, proximal limbs and trunk in a chronic, relapsing fashion. It is sometimes associated with overlying discoid lupus erythematosus, and even systemic lupus erythematosus. ${ }^{3}$ Histology shows a lobular panniculitis with characteristic eosinophilic hyaline fat necrosis.

This patient's constitutional symptoms could also raise suspicion of SPTCL, an indolent type of non-Hodgkin lymphoma, which can present like a panniculitis with deeply seated nodules on the legs and trunk. Histology for SPTCL would show atypical lymphocytes with hyperchromatic nuclei rimming adipocytes. $^{4}$

Our patient was diagnosed with pancreatic panniculitis following the skin biopsy, which showed characteristic features: a lobular panniculitis with extensive necrosis, an acute neutrophilic inflammatory infiltrate with characteristic ghost cells, and basophilic calcification. This was further supported by laboratory findings of raised lipase levels and radiological evidence of extensive mass lesions with pancreatic involvement. An eventual biopsy of one of the peritoneal masses confirmed a diagnosis of metastatic primary pancreatic adenocarcinoma.

Pancreatic panniculitis is uncommon, but harbours diagnostic significance as an early sign of pancreatic disease. Various pancreatic disorders have been associated with panniculitis, namely acute or chronic inflammatory pancreatitis, pancreatic carcinoma of different subtypes and metastatic cancer-causing pancreatic invasion. ${ }^{5}$

The pathogenesis remains unclear but is likely to be closely related to the systemic release of pancreatic enzymes - most clearly lipase, followed by amylase and trypsin-resulting in subcutaneous lipolysis. Other proposed mechanisms consider the role of traumatic, infective or trypsin-induced vascular damage, the special lipid conformations in adipocyte cell membranes increasing pancreatic enzyme absorption, adipokine release, immune complex formation and alpha-1antitrypsin deficiency in causing adipocyte necrosis and panniculitis..$^{5-7}$

Clinically, it presents as erythematous, oedematous, painful nodules on the lower extremities, sometimes on the anterior trunk, arms and scalp. ${ }^{6}$ They can occur singly or in crops, with the potential to ulcerate and migrate. ${ }^{6}$ Laboratory investigations often reveal raised lipase levels as in our patient's case. Tumour markers such as CA 19-9 may also be raised, ${ }^{8}$ as in this case.

Of note, our patient's presentation was accompanied by initial peripheral eosinophilia and inflammatory arthritis. This could represent fulfilment of Schmid's triad - a rare clinical syndrome characterised by a triad of panniculitis, polyarthritis and eosinophilia in patients 
with pancreatic tumour. This triad is associated with poorer prognosis. ${ }^{6,9}$

Management centres on treatment of the underlying pancreatic disease, such as resection of tumours, though supportive measures such as local compression may be helpful if ulcerations are present. Octreotide can also be considered in the setting of acute pancreatitis to inhibit pancreatic enzyme synthesis. ${ }^{6}$ Our patient underwent chemotherapy and follow-up scans showed radiological improvement of the peritoneal masses. Correspondingly, she had marked amelioration of her bilateral lower limb nodules, with reduced pain and some even resolving completely.

\section{REFERENCES}

1. Ishiguro N, Kawashima M. Cutaneous polyarteritis nodosa: A report of 16 cases with clinical and histopathological analysis and a review of the published work. The J Dermatol 2010;37:85-93.

2. Fernandez-Faith E, McDonnell J. Cutaneous sarcoidosis: Differential diagnosis. Clin Dermatol 2007;25:276-87.

3. Wick MR. Panniculitis: A summary. Semin Diagn Pathol 2017; 34:261-72.

4. Guitart J. Subcutaneous lymphoma and related conditions. Dermatol Therap 2010;23:350-5.
5. Yamashita $\mathrm{Y}$, Joshita $\mathrm{S}$, Ito $\mathrm{T}$, et al. A case report of pancreatic panniculitis due to acute pancreatitis with intraductal papillary mucinous neoplasm. BMC Gastroenterol 2020;20:286

6. Heykarts B, Anseeuw M, Degreef H. Panniculitis caused by acinous pancreatic carcinoma. Dermatology 1999;198:182-3.

7. Cutlan RT, Wesche WA, Jenkins JJ, et al. A fatal case of pancreatic panniculitis presenting in a young patient with systemic lupus. J Cutan Pathol 2000;27:466-71.

8. Zhang G, Cao Z, Yang G, et al. Pancreatic panniculitis associated with pancreatic carcinoma. Medicine (Baltimore) 2016;95:e4374.

9. Schmid M. The syndrome of metastasizing, exocrine pancreas adenoma secretory activity. Z Klin Med 1957;154:439-55.

Yihao Abraham Sui ${ }^{1}$, Wei Lien Rita Lai ${ }^{2}{ }^{M R C P}(U K)$, Weiting Michelle Liang ${ }^{3} M R C P(U K)$,

Ziying Vanessa $\underline{\operatorname{Lim}}^{3}{ }_{M R C P(U K)}$

${ }^{1}$ Yong Loo Lin School of Medicine, National University of Singapore, Singapore

${ }^{2}$ Internal Medicine, National Healthcare Group, Singapore

${ }^{3}$ National Skin Centre, Singapore

Correspondence: Dr Ziying Vanessa Lim, National Skin Centre, 1 Mandalay Road, Singapore 308205.

Email: vlim@nsc.com.sg 